\title{
Improving Information to Enable Effective Tobacco Control Intervention and Measure Progress Over Time: The Cambodia Country Experience
}

\author{
James Rarick ${ }^{1,2}$, They Kheam ${ }^{3}$, Yel Daravuth ${ }^{4}$, Edouard Tursan d'Espaignet ${ }^{5}$, \\ Mina Kashiwabara $^{6 *}$, Chhea Chhordaphea ${ }^{7}$, Bungon Ritthiphakdee ${ }^{8,9}$
}

\begin{abstract}
Tobacco is a leading cause of noncommunicable diseases (NCDs) which kill about 41 million people each year. Of these, 15 million die prematurely between the ages of 30 and 69 years, most of which occur in low-and middle-income countries (LMICs). The adoption of the Sustainable Development Goals and their targets under the 2030 Agenda for Sustainable Development provides a new impetus for countries to accelerate tobacco control efforts as they specifically call for strengthening implementation of the World Health Organization (WHO) Framework Convention on Tobacco Control and striving to achieve a one-third reduction in premature deaths from NCDs. While NCD prevention and control is a priority in the national strategic plans and policies for health in most countries in the Western Pacific Region, few have formally adopted a national target for reducing tobacco use. Article 20 of the WHO FCTC calls on all countries to improve tobacco surveillance to enable monitoring and evaluation of tobacco control efforts. The increase in timely and standardized comparable data presents new opportunities to set scientifically valid and achievable national indicators and targets for development and implementation of strong tobacco control measures. Cambodia is yet to establish national targets and full implementation of legislative measures. However, with strong tobacco surveillance mechanism in place, it can provide the country experience for a LMIC that has developed its own capacity to conduct periodic monitoring and surveillance of tobacco use and for using national data to advocate successfully for stronger tobacco control policies.
\end{abstract}

Keywords: Tobacco- noncommunicable diseases- primary prevention- health policy- public health surveillance

Asian Pac J Cancer Prev, 21, Progress of Tobacco Control in the Western Pacific Region Suppl, 5-8

\section{Introduction}

Noncommunicable diseases (NCDs) kill about 41 million people each year, accounting for more than $70 \%$ of all deaths in the world. Fifteen million of these deaths occur prematurely between the ages of 30 and 69 years and $85 \%$ in low- and middle-income countries (LMICs) (WHO, 2018). Tobacco use, recognized as a leading cause of NCDs, alone kills more than 8 million people each year (Institute for Health Metrics and Evaluation, 2019).

The Government of Cambodia endorsed the 2030 Agenda for Sustainable Development in 2015. The adoption of the Sustainable Development Goals (SDGs) and their targets provides a new impetus for countries to accelerate tobacco control efforts. To implement the SDGs, countries have called for strengthening implementation of the World Health Organization (WHO) Framework
Convention on Tobacco Control (FCTC). Article 20 of the Convention calls on all countries to improve tobacco surveillance to enable monitoring and evaluation of tobacco control efforts (WHO, 2003).

To encourage comparability across countries, WHO, the US Centers for Disease Control and Prevention (CDC) and Canadian Public Health Association partnered to set up the Global Tobacco Surveillance System (GTSS) in 1998 (Global Tobacco Surveillance System Collaborating Group, 2006). The initial focus of the GTSS was on youth through the Global Youth Tobacco Survey. In 2008, WHO and CDC expanded the GTSS to include adults in the Global Adult Tobacco Survey (GATS) - a household survey of people aged 15 years and over (Palipudi et al, 2016). The GTSS surveys use a standard protocol for sample design, questionnaire, field implementation, data collection, aggregation, analysis and reporting of results. 
In 2011, WHO and the US Centers for Disease Control and Prevention released the "Tobacco Questions for Surveys" (TQS) - a subset of questions from the Global Adult Tobacco Survey covering both tobacco use and tobacco control measures. The TQS has since increasingly been incorporated in part or in full into other survey systems, such as the WHO STEPwise multi-risk factor survey for noncommunicable diseases and the Demographic and Health Survey. The increase in timely and standardized comparable data presents new opportunities for all countries to develop and implement strong tobacco control measures, as well as setting scientifically valid and achievable national indicators and reduction targets.

While much progress in tobacco control surveillance has been made in all WHO regions, the information base needed for informed decision making is still relatively weak. There is an urgent need for countries to fund and implement regular populations-based surveys. Cambodia is one country in the WHO Western Pacific Region that has made substantial efforts over the past decade to improve its information base.

\section{Using data for policy development and intervention}

After Cambodia ratified the WHO FCTC in 2005, its first step was to assess the country's strengths and weaknesses in relations to selected tobacco control demand reduction measures. This assessment, captured in the WHO Report on the Global Tobacco Epidemic, indicated that Cambodia had relatively weak measures such as a smoke-free laws covering only limited types of public places and no policy on tobacco advertising bans (WHO, 2008). Efforts to revise Cambodia's tobacco control law began thereafter. There was, however, strong interference from the local and international tobacco industries to block development of stronger tobacco control measures (Southeast Asia Tobacco Control Alliance and Health Justice, 2015). Cambodia soon realized that it could not overcome this interference without better evidence to convince decision makers to take strong action.

\section{Development of new information}

Cambodia has been collecting adult tobacco use data since 2000 (Table 1). It undertook a Demographic Health Survey with questions on tobacco use in 2000 , a smoking behavior survey in 2004, and a tobacco use and religious beliefs survey in 2005/6. However, none of those surveys used standard validated questions and therefore produced limited information for decision making. Cambodia then conducted two Demographic and Health Surveys in 2010 and 2014. Those surveys contained information on tobacco prevalence but were limited to women aged 15-49 years of age and provided limited information on determinants of tobacco use that could inform tobacco reduction interventions.

To strengthen tobacco surveillance, the Government of Cambodia invested its own funds and expertise and also partnered with outside organizations such as South East Asia Tobacco Control Alliance (SEATCA), Loma Linda University and WHO for additional financial and technical resources. Through these partnerships, Cambodia carried out four national surveys which generated comprehensive data on tobacco epidemic in the country. Cambodia conducted its first NCD multi-risk factor survey, Cambodia STEPS Survey, in 2010, using globally standardized questions on NCD risk factors, with the nationally representative sample of men and women aged 25-64 years (University of Health Sciences and Ministry of Health, 2010). The STEPs survey at that time did not yet contain policy related questions. In 2011, Cambodia conducted its first National Adult Tobacco Survey of Cambodia (NATSC) which used globally standardized questions on tobacco use and demand reduction measures from GATS, and covered men and women aged 15 years and older (National Institute of Statistics, 2015). A repeat NATSC was carried out in 2014 (National Institute of Statistics, 2015). Cambodia also repeated the Cambodia STEPS survey in 2016, with an expanded age range of 18-69 compared to the first STEPS survey (University of Health Sciences and Ministry of Health, 2018). Despite the variance in the age ranges used in these surveys, the two NATSC and the two STEPS surveys, all using the standardized questions on tobacco use, provide comparable data for analyzing tobacco use trends for Cambodian adults over time.

The availability of comprehensive scientifically valid information collected from the NATSC and Cambodia STEPS surveys enabled the tobacco control community to effectively counter tobacco industry interference. These survey results were used to advocate for an increase in taxation on tobacco products, to mandate smoke-free

Table 1. National Aadult Surveys that Included Tobacco Use Indicators and Reported Smoking Rates since 2000

\begin{tabular}{|c|c|c|c|c|}
\hline \multirow[t]{2}{*}{ Survey name } & \multirow[t]{2}{*}{ Year } & \multirow[t]{2}{*}{ Age } & \multicolumn{2}{|c|}{ Current smoking } \\
\hline & & & Male & Female \\
\hline Cambodia STEPS Survey & 2016 & $18-69$ & 37.2 & 2.9 \\
\hline Demographic and Health Survey & 2014 & $15-49$ & $31.8^{*}$ & $2.3^{*}$ \\
\hline National Adult Tobacco Survey of Cambodia & 2014 & $15+$ & 32.9 & 2.4 \\
\hline National Adult Tobacco Survey of Cambodia & 2011 & $15+$ & 39.1 & 3.4 \\
\hline Cambodia STEPS Survey & 2010 & $25-64$ & 54.1 & 5.9 \\
\hline Demographic and Health Survey & 2010 & $15-49$ & 34.3 & 2.4 \\
\hline Demographic and Health Survey & 2005 & $15-49$ & NA & $3.6^{*}$ \\
\hline Smoking Behavior Survey & 2004 & $15+$ & 44.1 & 5.1 \\
\hline Demographic and Health Survey & 2000 & $15-49$ & NA & $10.5^{*}$ \\
\hline
\end{tabular}




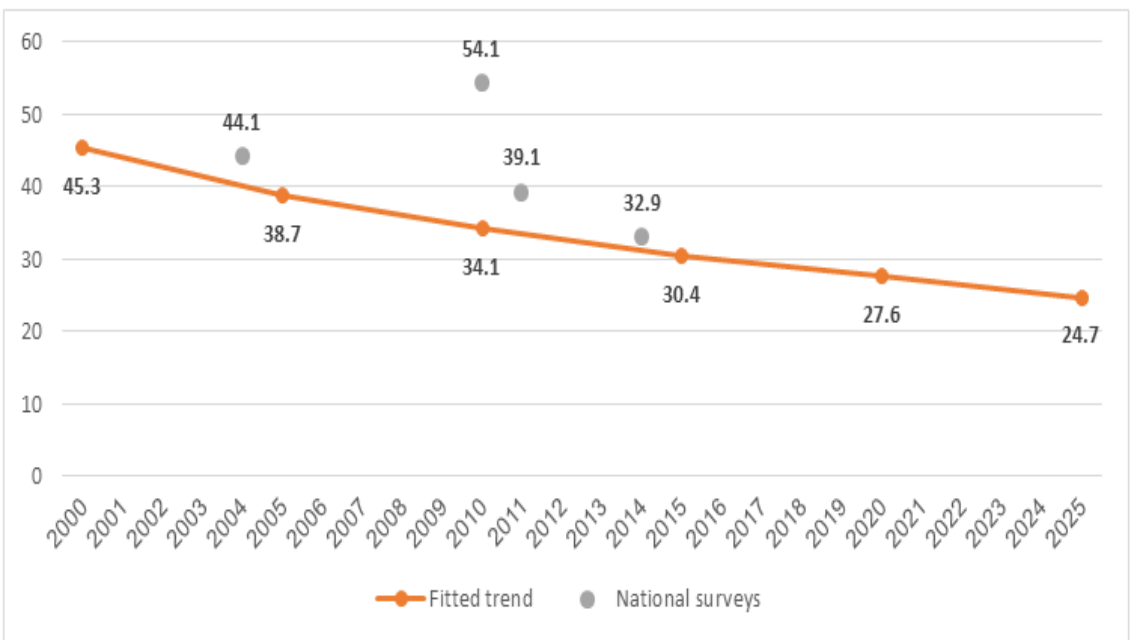

Figure 1. Fitted Trends in Current Tobacco Smoking among Men Aged $\geq 15$ Years, Cambodia. Source: WHO global report on trends in prevalence of tobacco use 2000-2025, third edition.

public places, implement pictorial health warnings on tobacco packaging, and to strengthen measures to restrict tobacco advertising and promotion. The survey data also indicated public support for strong tobacco control policies. A related policy paper was presented to the Council of Ministers, the National Assembly, and the Senate. These efforts led to successful adoption of comprehensive tobacco control measures including the 2015 Tobacco Products Control Law.

Taking the next step - Measuring progress over time

WHO began reporting estimates of the prevalence of tobacco smoking for all countries in the first report on the global tobacco epidemic, in 2008 (WHO, 2008). As the GTSS has generated a greater quantity of standardized data from countries, this has enabled WHO to develop a statistical tool to produce observed and projected trends in tobacco use. WHO has published three editions of these trends in the WHO global report on trends in prevalence of tobacco use 2000-2025 which provides country-by-country analysis of trend projections in tobacco smoking (WHO, 2015; WHO, 2018; WHO, 2019b). WHO used all available Cambodia data (except for the 2016 Cambodia STEPs survey as results were not available at time of producing the trends) to generate an underlying tobacco smoking trend for Cambodia (Figure 1).

Based on the projections, Cambodia will not achieve the voluntary global target of $30 \%$ relative reduction of tobacco use between 2010 and 2025 with business as usual. This projection points to a need for more intensive tobacco control efforts to protect the health of Cambodians.

An important advantage of timely and regular tobacco surveillance data is that these provide Cambodian authorities and tobacco control partners with a capacity to project the impact of interventions on future tobacco use. This information can be a potentially powerful tool for engaging policy-makers and the general public in the process of setting national targets and agreeing on future action to reach those targets.
Partly as a result of improving its tobacco control surveillance systems, Cambodia has been able to adopt stronger tobacco control measures and has begun to develop its regulatory framework through the adoption of several sub-decrees relating to enforcement of the existing tobacco control laws. An additional benefit is that Cambodia has been designated as one of the world's first FCTC 2030 countries - selected LMICs receiving direct support from the WHO FCTC Secretariat, the United Nations Development Programme (UNDP) and WHO Department of Prevention of Noncommunicable Diseases in accelerating the implementation of the WHO FCTC . This has led to the development of an investment case for tobacco control in Cambodia, launched in 2019 (RTI International, Ministry of Health of Cambodia, United Nations Development Programme, WHO FCTC Secretariat and World Health Organization, 2019). This investment case anticipates significant benefits ranging from saving thousands of lives to preventing economic loss from tobacco activities. With a strong sustained capacity for conducting monitoring and surveillance, Cambodia will be able to take corrective and new actions when necessary to protect its population from harm.

\section{Acknowledgments}

\section{Disclaimer}

The authors alone are responsible for the views expressed in this article and they do not necessarily represent the views, decisions or policies of the institutions with which they are affiliated.

Statement conflict of Interest

The authors confirm that there are no known conflicts of interest associated with this publication and there has been no significant financial support for this work that could have influenced its outcome.

\section{References}

Global Tobacco Surveillance System Collaborating Group (2006). Global Tobacco Surveillance System (GTSS): 
Purpose, Production, and Potential. J Sch Health, 75, 15-24.

Institute for Health Metrics and Evaluation (2019). Global Burden of Disease. https://vizhub.healthdata.org/gbdcompare/.

National Institute of Statistics (2011). 2011 National Adult Tobacco Survey of Cambodia (NATSC, 2011). Ministry of Planning, Phnom Penh, Cambodia.

National Institute of Statistics (2015). 2014 National Adult Tobacco Survey of Cambodia (NATSC, 2011). Ministry of Planning, Phnom Penh, Cambodia.

Palipudi KM, Morton J, et al (2016). Methodology of the Global Adult Tobacco Survey - 2008-2010. Glob Health Promot, 23, 3-23.

RTI International, Ministry of Health of Cambodia, United Nations Development Programme, WHO FCTC Secretariat and World Health Organization (2019). Investment Case for Tobacco Control in Cambodia: The case for scaling-up WHO FCTC implementation. United Nations Development Programme, New York, USA.

Southeast Asia Tobacco Control Alliance and Health Justice (2015). Preventing Tobacco Industry Interference: A Toolkit for Advocates and Policymakers. Southeast Asia Tobacco Control Alliance, Bangkok, Thailand; and Health Justice, Quezon City, Philippines.

University of Health Sciences and the Preventive Medicine Department of Ministry of Health (2010). Prevalence of Non-communicable Disease Risk Factors in Cambodia: STEPS Survey Country Report. University of Health Sciences and Ministry of Health, Phnom Penh, Cambodia.

University of Health Sciences and the Preventive Medicine Department of Ministry of Health (2018). Prevalence of Non-communicable Disease Risk Factors in Cambodia: STEPS Survey Country Report. University of Health Sciences and Ministry of Health, Phnom Penh, Cambodia.

World Health Organization (2015). WHO global report on trends in prevalence of tobacco smoking 2000-2025 - First edition. World Health Organization, Geneva, Switzerland.

World Health Organization (2003). WHO Framework Convention on Tobacco Control. World Health Organization, Geneva, Switzerland.

World Health Organization (2008). WHO report on the global tobacco epidemic, 2008. World Health Organization, Geneva, Switzerland.

World Health Organization (2018). Noncommunicable diseases fact sheet. https://www.who.int/news-room/fact-sheets/ detail/noncommunicable-diseases.

World Health Organization (2018). WHO global report on trends in prevalence of tobacco smoking 2000-2025 - Second edition. World Health Organization, Geneva, Switzerland.

World Health Organization (2019a). WHO report on the global tobacco epidemic, 2019. World Health Organization, Geneva, Switzerland.

World Health Organization (2019b). WHO global report on trends in prevalence of tobacco use 2000-2025, third edition. World Health Organization, Geneva, Switzerland.

This work is licensed under a Creative Commons AttributionNon Commercial 4.0 International License. 